\title{
Comment on: "Hepatoid adenocarcinoma of the stomach: a unique subgroup with distinct clinicopathological and molecular features. Gastric Cancer, 2019: 1-10" by Wang et al.
}

\author{
Chaoran $\mathrm{Yu}^{1,2}$ (D) \\ Received: 27 May 2019 / Accepted: 16 August 2019 / Published online: 23 August 2019 \\ (c) The International Gastric Cancer Association and The Japanese Gastric Cancer Association 2019
}

\section{Dear Editor,}

In a Chinese study analyzing the clinicopathological and molecular characteristics of hepatoid adenocarcinoma of stomach (HAS), Wang et al. identified a list of frequently mutated genes and highlighted the prognostic value of preoperative serum AFP level associated with HAS [1]. To the best knowledge, this is the largest genomic study upon Chinese HAS patients. However, some issues need to be addressed.

Firstly, Wang et al. indicated that HAS was not classified as any of four subtypes in the cancer genome atlas (TCGA) due to the lack of morphological evidence [1]. In fact, the four genomic subtypes defined by TCGA are based on six molecular platforms, including somatic copy number array, exome sequencing, DNA methylation array, RNA sequencing, miRNA sequencing and reverse-phase protein array, regardless of morphological features [2]. Reasonably presume, the exact TCGA subset which HAS belongs to could also be determined using similar pattern, instead of morphological matching.

By morphological definition, hepatoid carcinoma (type I) is defined as unequivocal morphological evidence of hepatocellular differentiation, resembling normal hepatocytes. Interestingly, in stomach adenocarcinoma (STAD) of TCGA, 20 cases display elevated AFP expression with inferior survival [3]. However, none of the cases in STAD is morphologically identified as HAS [3]. In fact, those elevated-AFP cases are marked as tubular category by WHO classification [3]. Of note, elevated-AFP cases in STAD are categorized as

Chaoran $\mathrm{Yu}$

chaoran_yu@yeah.net

1 Fudan University Shanghai Cancer Center, Shanghai 200025, People's Republic of China

2 Department of Oncology, Shanghai Medical College, Fudan University, Shanghai 200025, People's Republic of China chromosomal instability (CIN) subtypes with microsatellite instability stable (MSS), of which TP53 is one of the most significantly mutated genes [2]. Similarly, TP53 was also the top mutated genes and all 24 HAS cases were MSS in Wang et al. [1]. Meanwhile, the cases with elevated preoperative serum AFP in Wang et al. were also significantly associated with poorer overall survival [1]. Therefore, it is reasonably to speculate that HAS belongs to the CIN subset of TCGA which, however, warrants further validation.

Secondly, Wang et al. reported that HAS patients with low serum AFP level $(<500 \mathrm{ng} / \mathrm{ml}, n=14)$ indeed had a significantly better outcome than patients with high serum AFP $(n=10)$. However, the clinicopathological characterization between HAS patients with high/low serum AFP level were not displayed, which may confound final outcome. In addition, given the fact that HAS patients are prone to develop liver metastasis, reasonably, HAS tumor progression could be dynamically reflected by the serum AFP level both in preoperative and postoperative courses. In fact, the followup value of serum AFP has not been fully recognized.

\section{References}

1. Wang Y, Sun L, Li Z, et al. Hepatoid adenocarcinoma of the stomach: a unique subgroup with distinct clinicopathological and molecular features. Gastric Cancer. 2019. https://doi.org/10.1007/ s10120-019-00965-5.

2. Cancer Genome Atlas Research Network. Comprehensive molecular characterization of gastric adenocarcinoma. Nature. 2014;513(7517):202.

3. Arora K, Bal M, Shih A, et al. Fetal-type gastrointestinal adenocarcinoma: a morphologically distinct entity with unfavourable prognosis. J Clin Pathol. 2018;71(3):221-7.

Publisher's Note Springer Nature remains neutral with regard to jurisdictional claims in published maps and institutional affiliations. 